\title{
INFLUÊNCIA DOS NIVEIS DE ANSIEDADE, DEPRESSÃO E DOR NA QUALIDADE DE VIDA DE PACIENTES EM UCO DE UM HOSPITAL
}

Natalia Faria Rinaldi, Daiane Caroline Nascimento dos Santos, Layane Lopes Napoleão, Renata Calciolari Rossi e Silva, Susimary Aparecida Trevizan Padulla

${ }^{1}$ Discente do Curso de Graduação de Fisioterapia - FCT/UNESP - Presidente Prudente. ${ }^{2}$ Discente do Curso de Pós Graduação em Fisioterapia - FCT/UNESP. ${ }^{3}$ Docente do Curso de Graduação em Fisioterapia - FCT/UNESP - Presidente Prudente. ${ }^{4}$ Docente Orientadora do Curso de Graduação em Fisioterapia - FCT/UNESP - Presidente Prudente. E-mail: nataliafariar@gmail.com

\section{RESUMO}

A unidade de terapia intensiva coronariana (UCO) é um setor de alta complexidade que destina-se a pacientes cardiopatas graves, sendo atribuída uma imagem atrelada ao sentimento de medo, sobretudo da morte, devido a isso acredita-se que há altos níveis de ansiedade, depressão e dor, o que pode influenciar na qualidade de vida. Assim, o objetivo deste estudo é identificar os níveis de ansiedade, depressão, dor e qualidade de vida de pacientes internados em uma UCO de um hospital público. Foram selecionados indivíduos internados na UCO, maiores de 18 anos, que estivessem conscientes. Foram coletados dados de perfil sóciodemográfico, no momento da entrevista, os pacientes irão responder aos questionários de ansiedade e depressão (HAD) e de qualidade de vida (WHOQOL-BREF), e identificaram seu nível de dor por meio da escala visual analógica (EVA). Os resultados obtidos foram média de idade 61,92. A média revelou presença de ansiedade, porém não de depressão, assim como os domínios da QV mais afetados foram a saúde psicológica e a relação social.

Palavras-chave: unidades de terapia intensiva; coronariano; ansiedade; depressão; qualidade de vida.

\section{INTRODUÇÃO}

A unidade de terapia intensiva coronariana (UCO) é uma unidade específica para procedimentos e patologias cardíacas com uso de tecnologias relacionadas a tal sistema, que destina-se a pacientes cardiopatas graves e, como tal, pode ser atribuída à UCO uma imagem atrelada ao sentimento de medo, sobretudo da morte ${ }^{1-3}$.

No início do intensivismo, os pacientes morriam em 24 horas, tendo a UTI relação direta com a morte, dor e desespero. Atualmente, este setor do complexo hospitalar tem condições de oferecer maiores chances de recuperação ao paciente, para que este restabeleça o equilíbrio orgânico, dando continuidade ao tratamento ${ }^{4-6}$.

Os fatores de risco considerados modificáveis que podem agravar o quadro de pacientes cardíacos internados em UCO, são, o medo de morrer, a ansiedade e a depressão, que são mais acentuados, pois este local é normalmente associado a um perigo potencialmente maior ${ }^{6,10}$.

A ansiedade e depressão estão diretamente relacionadas ao desenvolvimento da doença 
coronariana e ao seu respectivo prognóstico, porém permanece incerto o significado clínico desta associação ${ }^{9,12}$.

Além desses aspetos, a dor é outro fator pode agravar o quadro de pacientes cardiopatas, principalmente quando alocados em UCO, podendo ser determinante para manutenção do paciente nesta unidade. Dor é referida como o quinto sinal vital e é uma importante razão pela qual os pacientes procuram cuidados de saúde ${ }^{13}$. Durante a hospitalização, a dor é frequente, isso ocorre devido as graves doenças presentes e aos procedimentos necessários ao tratamento.

Estudos recentes sobre QV têm sido realizados em pacientes portadores de várias condições mórbidas objetivando melhorar a escolha das medidas terapêuticas, e como consequência, visando estimular positivamente as condições clínicas desses pacientes ${ }^{7,8,14}$.

A opção por estudar estes aspectos ansiedade, depressão e dor em pacientes em UCO, justifica-se pelo fato de que identificar a presença ou ausência destes deve facilitar o delineamento de estratégias terapêuticas voltadas para a melhora dos pacientes, ou seja, em todos os aspectos a esta relacionada.

Hipotetiza-se que haverá presença de ansiedade, depressão e dor, e que quanto maior os níveis desses aspectos, menores serão a QV, ou seja, se há uma relação inversamente proporcional.

\section{OBJETIVOS}

Identificar os níveis de ansiedade, depressão, dor e qualidade de vida de pacientes internados em uma UCO de um hospital público.

\section{MATERIAIS E MÉTODO}

Estudo observacional prospectivo ${ }^{20}$. Foram admitidos pacientes adultos, de ambos os sexos internados na unidade de terapia intensiva coronariana (UCO), portadores de diversas patologias cardíacas ou submetidos a procedimentos cardíacos.

Serão excluídos pacientes compreedidos na faixa etária neonatal e infanto-juvenil, pacientes que se encontrarem inconcientes, pacientes que não possuírem patologia cardíaca, ou que não serão submetidos a procedimentos cardíacos.

O estudo foi realizado na UCO da Santa Casa de Misericórdia na cidade de Presidente Prudente - SP.

Foram aplicados questionários específicos para avaliação das variáveis de desfecho, tais 
como: a Escala Hospitalar de Ansiedade e Depressão (HAD), a Escala Visual Analógica (EVA) e o Questionário de Qualidade de Vida World Health Organization Quality of Life (WHOQOL-Bref).

A Escala Hospitalar de Ansiedade e Depressão (Hospital Anxiety and Depression Scale HADS), validada e traduzida para a língua portuguesa, é constituída de duas subescalas, sendo uma para ansiedade e outra para depresão. Cada subescala é composta por sete questões de múltipla escolha. A resposta de cada item esta compreendido numa escala ordinal de quatro pontos (0 inexistente a 3 - muito grave) ${ }^{11,21-23}$. O escore total do HAD varia de 0 a 21 pontos (sendo 0 a 21 para ansiedade e 0 a 21 para depressão) $)^{22-23}$.

A escala visual analógica (EVA) é uma linha de $10 \mathrm{~cm}$ de comprimento, aonde cada extremidade da linha descreve, nenhuma dor graduando até a pior dor imaginável.

A qualidade de vida foi avaliada através do WHOQOL-BREF, que consiste em 26 itens com abragência em quatro domínios: saúde física, saúde psicológica, relação social e meio ambiente. Os escores dos domínios são calculados a partir dos itens e depois convertidos para uma escala percentual global que varia de muito ruim (0\%) a muito boa $(100 \%)^{25-27}$.

Os pacientes realizaram a leitura e assinaram um Termo de Consentimento Livre e Esclarecido. O estudo foi aprovado pelo Comitê de Ética e Pesquisa da Universidade Estadual Paulista "Júlio De Mesquita Filho" campus Presidente Prudente-SP processo de no 23/2010.

\section{RESULTADOS}

O indicador social referente ao gênero, demonstra que de 56 pacientes, 18 eram mulheres e 38 eram homens, sendo que destes $3(5,4 \%)$ eram solteiros, 3 (5,4\%) eram divorciados, 8 (14,3\%) eram viúvos e 42 (75\%) eram casados.

Por meio da escala de ansiedade e depressão HAD, foram verificadas as médias da ansiedade e da depressão dos pacientes internados na UCO. (Tabela 1).

Hospital Anxiety and Depression Scale - HAD

HAD Ansiedade Depressão

\begin{tabular}{lll}
\hline \hline Média & 10,21 & 9,32 \\
\hline Tabela 1 &
\end{tabular}

Através da escala visual analógica (EVA) a dor foi avaliada, revelando uma média de 1,6, 
numa escala de 0 a 10.

Por meio do questionário de qualidade de vida WHOQOL-BREF, foram verificadas os quatro domínios, dos pacientes internados na UCO. (Gráfico 1).

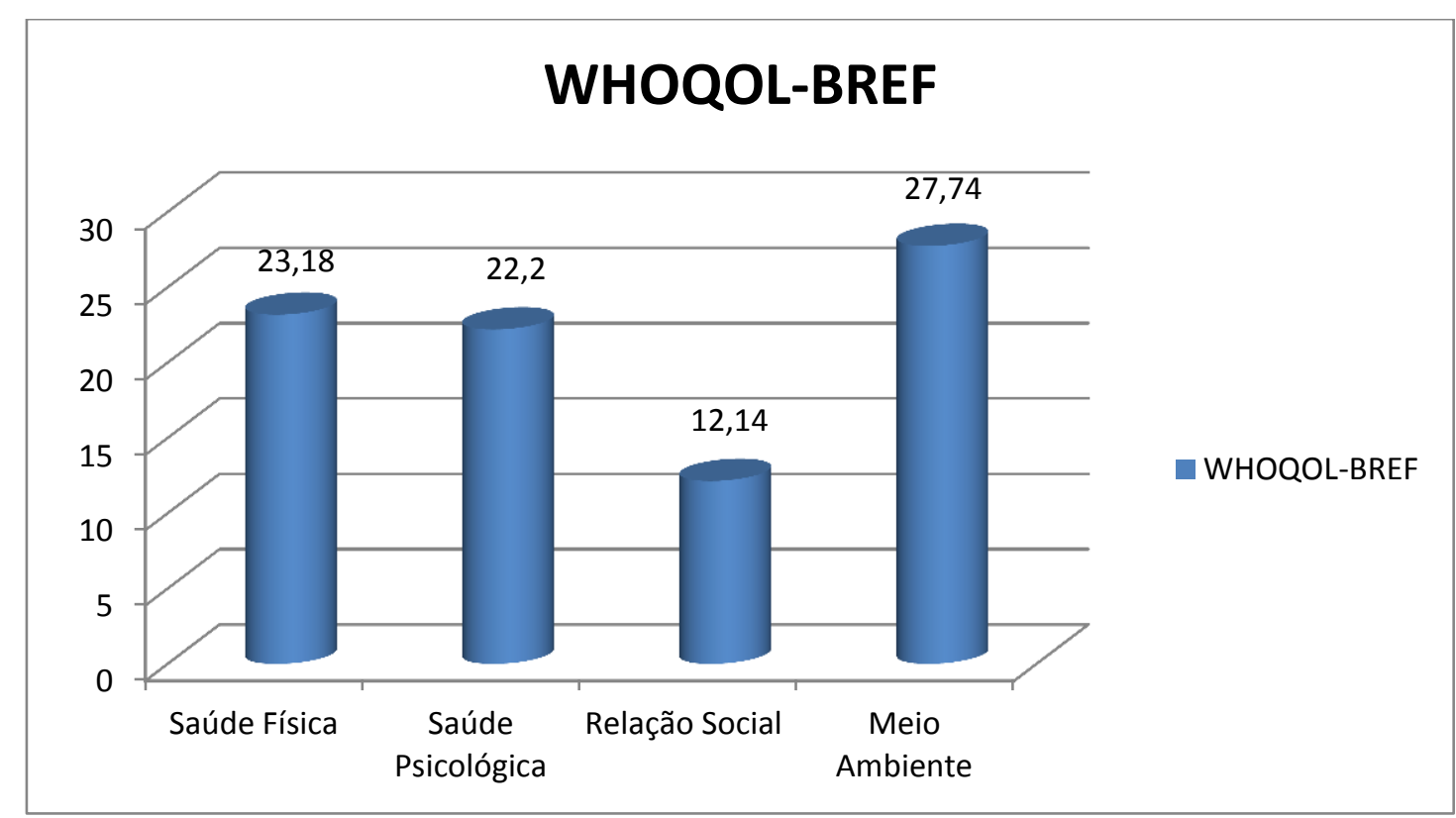

Gráfico 1. Qualidade de vida medida no momento da entrevista.

\section{DISCUSSÃO}

Sabe-se que a unidade de terapia intensiva coronariana (UCO) destina-se a pacientes cardiopatas graves e, como tal, pode ser atribuída à UCO uma imagem atrelada ao sentimento de medo, sobretudo da morte ${ }^{1-3}$. Esses sentimentos interferem diretamente na ansiedade $\mathrm{e}$ depressão dos pacientes, e a ansiedade e depressão por sua vez, estão diretamente relacionadas ao desenvolvimento da doença coronariana e ao seu respectivo prognóstico. ${ }^{9,12}$

Dor é referida como o quinto sinal vital e é uma importante razão pela qual os pacientes procuram cuidados de saúde ${ }^{13}$, durante a internação e sua permanência na UCO a abordagem terapêutica de pacientes gravemente enfermos são quase sempre invasivos e agressivos, principalmente nos setores de emergência ${ }^{15-17}$, contudo devido os cuidados e provavelmente aos medicamentos sedativos a dor se revelou pequena neste estudo.

Os aspectos citados anteriormente interferem diretamente na QV dos pacientes internados em UCO, o cuidado intensivo deve ter como objetivo não só o diagnóstico e o tratamento das doenças, mas a restauração da condição de saúde e da QV prévia dos pacientes ${ }^{18-19}$, assim permitindo entender melhor quais os domínios mais afetados, com a tentativa futura de otimizar estes pontos. Na literatura, encontra-se trabalhos com o WHOQOL-Bref e seus achados mostram 
que os domínios que mais interferem negativamente na QV são a Saúde Psicológica e a Relação Social ${ }^{28-31}$,

Este estudo corrobora com os fatos, uma vez que foram os mesmos domínios mais afetados.

\section{CONCLUSÃO}

Em suma, houve presença de ansiedade nesta população, porém não houve dor significativa. A QV sofreu interferência direta na saúde psicológica e a relação social.

\section{REFERÊNCIAS}

1. Bento DCP, Prado APO, Gardenghi G. Estudo dos níveis de estresse dos pacientes submetidos à angioplastia durante a internação na unidade de terapia intensiva. Revista eletrônica de ciência e saúde. 2012;2(1):7-21.

2. Stumm EMF, Kuhn DT, Hildebrandt LM, Kirchner RM. Estressores vivenciados por pacientes emu ma UTI. Cogitare Enferm 2008;13(4):499-506.

3. Maciel ICF, Fernandes AC, Araújo TL. Unidade de Terapia Intensiva: sentimentos e expectativas quanto ao tratamento. Cad Centro Universit São Camilo. 2001;7(1):27-37.

4. Zobel $C$, Dörpinghaus $M, \underline{\text { Reuter } H}$, Erdmann E. Mortality in a cardiac intensive care unit. Clin Res Cardiol. 2012;101(7):521-4. http://dx.doi.org/10.1007/s00392-012-0421-9

5. Fontana CJ, Pittiglio LI. Sleep deprivation among critical care patients. Crit Care Nurs Q. 2010;33(1):75-81.

6. Cesarino CB, Rodrigues AMS, Mendonça RCHR, Corrêa LCL, Amorim RC. Percepções dos pacientes em relação à Unidade Terapia Intensiva. Arq Ciênc Saúde. 2005;12(3):158-61.

7. Takiut ME, Hueb W, Hiscoc,SB, Nogueira CRSR, Girardi P, Fernandes F, Favarato F, Lopes N, Borges JC, Góis AFT, Ramires JAF. Qualidade de Vida após Revascularização Cirúrgica do Miocárdio, Angioplastia ou Tratamento Clínico. Arq Bras Cardiol. 2007;88(5):537-544.

8. Soto M, Failde I, Marquez S, Benitez E, Ramos I, Barba A, et al. Physical and mental component summaries score of the SF-36 in coronary patients. Quality of Life Research. 2005;14:759-68.

9. Vasconcelos CB. Qualidade de vida, ansiedade e depressão após infarto do miocárdio. [dissertação]. Uberlândia: Universidade Federal de Uberlândia; 2007.

10. Bitencourt AG, Neves FBCS, Dantas MP, Albuquerque LC, Melo RMV, Almeida AM, et al. Análise de estressores para o paciente em unidade de terapia intensiva. Rev Bras Ter Intensiva. 2007;19(1):53-9. http://dx.doi.org/10.1590/S0103-507X2007000100007 
11. Correia DT, Barbosa A. Ansiedade e depressão em medicina. Modelos Teóricos e Avaliação. Acta Med Port. 2009;22(1):89-98.

12. Van Praag H, De Kloet RON, Van OSJ. Stress, the brain and depression. Cambrigde University Press. Cambridge. 2004;9:225-258.

13. Khatri A, Kalra N. A Comparison of Two Pain Scales in the Assessment of Dental Pain in East Delhi Children. International Scholarly Research Network. 2011.

14. Pocock SJ, Henderson RA, Clayton T, Lyman G, Chamberlain DA. Quality of life after coronary angioplasty or continued medical therapy for angina: threeyear follow-up in the RITA-2 Trial. J Am Coll Cardiol. 2000;35:907-14. http://dx.doi.org/10.1016/S0735-1097(99)00637-3

15. Viana DL, Dupas G, Pedreira MLG. A avaliação da dor da criança pelas enfermeiras na unidade de terapia intensiva. Pediatria (São Paulo) 2006;28(4):251-61.

16. Söderhamn O, Idvall E. Nurses' influence on quality of care in postoperative pain management: a phenomenological study. Int J Nurs Pract. 2003;9:26-32. http://dx.doi.org/10.1046/i.1440$\underline{172 X .2003 .00399 . x}$

17. Lynch M. Pain as the fifth vital sign. J intraven Nurs. 2001;24:85-94.

18. Angus DC, Carlet J. Surviving intensive care: a report from the 2002 brussels rountable. Intensive Care Med. 2003;29:368-77.

19. Black NA, Jenkinson C, Hayes JA, Young D, Vella K, Daly K. Review of outcome measures used in adult critical care. Crit Care Med. 2001;29:2119-24. http://dx.doi.org/10.1097/00003246$\underline{200111000-00012}$

20. Vest MT, Murphy TE, Araujo KLB, Pisani MA. Disability in activities of daily living, depression, and quality of life among older medical ICU survivors: a prospective cohort study. Health and Quality of Life Outcomes. 2011;9(9):1-10.

21. Jonston M, Pollard B, Hennessey P. Construct validation of the Hospital Anxiety and Depression Scale with clinical populations. J Psych Res. 2000;48(6):579-584. http://dx.doi.org/10.1016/S00223999(00)00102-1

22. Pais JR, Silva I, Ferreira T, Martins A, Meneses R, Baltar M. Validation study of a portuguese version of the hospital anxiety and depression scale. Psychol, Health Med 2007;12:225-237. http://dx.doi.org/10.1080/13548500500524088

23. Pfaffenberger $\mathrm{N}$, Doering $\mathrm{S}$, Puffinger $\mathrm{P}, \underline{\text { Höfer } \mathrm{S}}$, Alber $\mathrm{H}$, Ruttmann $\mathrm{E}$, Günther $\mathrm{V}$, Kopp $\mathrm{M}$. Health-related quality of life, anxiety and depression before and after coronary artery bypass grafting. Wien Med Wochenschr. 2010;160(1-2):44-53. http://dx.doi.org/10.1007/s10354-009$\underline{0722-4}$

24. Maruiti MR, Galdeano LE, Farah OGD. Ansiedade e depressão em familiares de pacientes internados em unidade de cuidados intensivos. Acta Paul Enferm 2008;21(4):636-42. http://dx.doi.org/10.1590/S0103-21002008000400016 
25. Tabah A, Philippart F, Timsit JF, Willems V, Français A, Leplège A, Carlet J, Bruel C, Misset B, Orgeas MG. Quality of life in patients aged 80 or over after ICU discharge. Critical Care. 2010;14(R2):1-7.

26. Power M, Quinn K, Schmidt S: Development of the WHOQOL-old module. Qual Life Res. 2005:14(1): 2197-2214. http://dx.doi.org/10.1007/s11136-005-7380-9

27. Skevington SM, Lotfy M, O'Connell KA: The World Health Organization's WHOQOL-BREF quality of life assessment: psychometric properties and results of the international field trial. A report from the WHOQOL group. Qual Life Res. 2004;13:299-310. http://dx.doi.org/10.1023/B:QURE.0000018486.91360.00

28. Chaimowicz F. A saúde dos idosos brasileiros às vésperas do século XXI: problemas, projeções e alternativas. Rev Saude Publica. 1997;31(2):184-200. http://dx.doi.org/10.1590/S0034$\underline{89101997000200014}$

29. Fleck MPA, Chachamovich E, Trentini CM. Projeto WHOQOL-OLD: método e resultados de grupos focais no Brasil. Rev Saude Publica. 2003;37(6):793-9. http://dx.doi.org/10.1590/S0034$\underline{89102003000600016}$

30. Lebrão ML, Laurenti R. Condições de saúde. In: Lebrão ML, Duarte YAO. SABE: saúde, bem-estar e envelhecimento. O Projeto SABE no município de São Paulo: uma abordagem inicial. Brasília: Organização Pan-Americana da Saúde; 2003. p. 73-91.

31. Organización Mundial de la Salud. Salud y envejecimiento: un documento para el debate. Versión preliminar. 2001;4:40. (Boletín sobre el Envejecimiento: Perfiles y Tendencias.) 